\title{
Photoluminescence Dynamics of GaN/Si Nanowires
}

\author{
K.P. Korona ${ }^{a}$, Z.R. Zytkiewicz ${ }^{b}$, P. Perkowska ${ }^{a}$, J. Borysiuk ${ }^{a, b}$, J. Binder $^{a}$, \\ M. SOBAnSKa ${ }^{b}$ And K. KLOSEK ${ }^{b}$ \\ ${ }^{a}$ Institute of Experimental Physics, Faculty of Physics, University of Warsaw, Hoża 69, 00-681 Warsaw, Poland \\ ${ }^{b}$ Institute of Physics, Polish Academy of Sciences, al. Lotników 32/46, 02-668 Warsaw, Poland \\ In this work we present analysis of carriers dynamics in samples of GaN nanowires grown on silicon. The \\ samples exhibit bright luminescence of bulk donor-bound excitons at $3.472 \mathrm{eV}$, surface defect-bound excitons \\ at $3.450 \mathrm{eV}$ (SDX) and a broad $(0.05 \mathrm{eV})$ band centered at $3.47 \mathrm{eV}$ caused probably by single free exciton and \\ bi-exciton recombination. The SDX emission has long lifetime $\tau=0.6 \mathrm{~ns}$ at $4 \mathrm{~K}$ and can be observed up to $50 \mathrm{~K}$. \\ At higher temperatures luminescence is dominated by free excitons. The broad excitonic band is best visible \\ under high excitation, and reveals fast, non-exponential dynamics. We present mathematical model assuming \\ exciton-exciton interaction leading to the Auger processes. The model includes $n^{2}$ (Langevin) term and describes \\ well the non-exponential dynamics of the excitonic band.
}

PACS: 78.55.Cr, 73.22.-f, 78.47.jd

\section{Introduction}

GaN is well-known for its excellent optoelectronic properties and excellent thermal stability. Structures based on GaN are playing an important role in the field of light emitting devices, photodetectors and sensors. Since a few years a new interest and growing number of publications [1-3] on GaN nanowires (NWs) are observed which is connected with development of growth techniques resulting in good quality, reproducible nanowires. This gives a hope for production of sensors and other devices based on the nanowires in the nearest future. For example, achievements on a coherent light source, also termed a polariton laser, have been lately reported [3].

Typical GaN/Si nanowires are a few tens of nanometers wide and a few hundreds nm long, so they do not show effects related to quantum confinement of electrons. However, they are small enough to influence carrier distribution and cause photon confinement and to change recombination dynamics. Here we present results showing interesting optical properties of nanowires resulting from exciton condensation in the nanowires (NWs).

\section{Experiment}

The GaN nanowires were grown at $\approx 740^{\circ} \mathrm{C}$ by plasma-assisted MBE on in situ nitridized $\mathrm{Si}(111)$ substrates. No catalyst was used for nucleation of NWs. The growth direction was [0001]. The high-resolution electron microscopy (see Fig. 1) reveals that the nanowires are nearly defect free and are well aligned with the $c$-axis being perpendicular to the substrate. The wires were about $400 \mathrm{~nm}$ long and $50 \mathrm{~nm}$ wide.
The time-resolved photoluminescence (TRPL) was measured with use of a $30 \mathrm{~cm}$ spectrograph $(0.05 \mathrm{~nm}$ resolution) and a synchroscan streak camera (2.5 ps resolution). The photoluminescence (PL) was excited by third harmonic of Ti:sapphire laser, tuned to $300 \mathrm{~nm}$. The pulses had time span of $130 \mathrm{fs}$ and frequency $80 \mathrm{MHz}$.

\section{Results and discussion}

The GaN nanowires exhibit very strong photoluminescence, not vanishing even at room temperature, which suggested good crystal quality and low number of defects. At low temperature a strong emission could be observed in the near bandgap region at energies from 3.3 to $3.5 \mathrm{eV}$. The defect-related yellow luminescence was very weak. One could distinguish bands well known from bulk GaN: free excitons (FX) at $3.48 \mathrm{eV}$, excitons bound to donors (DX) at $3.472 \mathrm{eV}$, excitons bound to acceptors (AX) $3.466 \mathrm{eV}$, and donor-acceptor transitions (DA) at $3.29 \mathrm{eV}$ (very weak). Widths of bound exciton peaks (DX and $\mathrm{AX}$ ) were between 1.5 and $3 \mathrm{meV}$.

The positions of these peaks were exactly the same as in unstrained homoepitaxial GaN samples [4]. Moreover, two extra features could be observed in the time-resolved PL spectra. The first one was a relatively narrow peak at $3.45 \mathrm{eV}$ that had a lifetime of about $0.6 \mathrm{~ns}$ (longer than DX lifetime $=0.4 \mathrm{~ns}$ ). This peak was reported in literature as characteristic for GaN nanowires [1] and related to excitons bound to surface defects (SDX) [2] or two-electron recombination of the DX near the surface [5]. The nanowires have a large area of side walls that are non-polar (1100) planes and probably can bind excitons. The second feature was short lived, broad $(0.05 \mathrm{eV})$ 
band centered at $3.47 \mathrm{eV}$ that can be interpreted as bi-exciton recombination or as free exciton recombination with Auger-type energy transfer to another exciton.

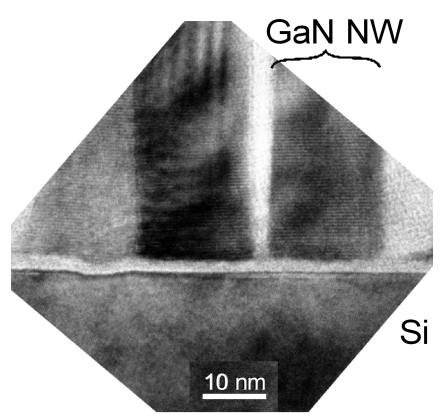

Fig. 1. High resolution transmission electron microscopy (HR TEM) image of the sample shows atomic layers inside the NWs.

During a short time after the laser pulse, the excitonic line is asymmetrically broadened. The high-energy wing has an exponential slope, which is marked in Fig. 2 with dashed line. The hot excitons have additional kinetic energy, so the emitted photons have higher energy and cause broadening of the high-energy wing of the FX peak. The characteristic exponential shape reflecting thermal distribution of excitons can be described by the following equation $[6,7]$ :

$$
I\left(\hbar \omega_{0}+E\right)=A E^{1 / 2} \exp \left(\frac{-E}{k_{\mathrm{B}} T_{\mathrm{X}}}\right),
$$

where $\hbar \omega_{0}+E$ is the exciton emission energy (including kinetic energy $E$ ), $T_{\mathrm{X}}$ - the exciton temperature and $A$ is the amplitude of the line. We can estimate $T_{\mathrm{X}}$ by the analysis of the shape of the excitonic peak. The initial $T_{\mathrm{X}}$ (at short time after excitation) was highest. Then excitons cooled down. The initial $T_{\mathrm{X}}$ logarithmically increased with excitation power density from $33 \mathrm{~K}$ at $0.01 \mathrm{~mW}$ up to $110 \mathrm{~K}$ at $10 \mathrm{~mW}$.

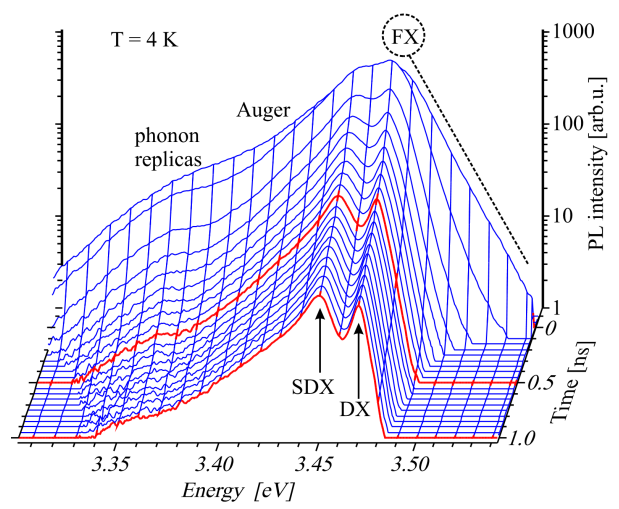

Fig. 2. Time-resolved PL spectra of the $\mathrm{GaN} / \mathrm{Si}$ nanowires.

In nanowires, we observed also asymmetric broadening of the low-energy side of the excitonic peak. This broadening can be explained assuming recombination of hot bi-excitons. In this case bi-exciton has momentum $p$ and kinetic energy $E_{\mathrm{kxx}}=p^{2} / 2 M$. When one exciton from the pair recombines, the other exciton in Auger-like process takes momentum of both, so it has energy $E_{\mathrm{kXX}}=p^{2} / M$, which means its energy is twice higher than initial energy. In such a case, instead of adding the kinetic energy to the photon (like in Eq. (1)), we should subtract it. That leads to the broadening of the low-energy wing.

Figure 3 shows power dependence of the luminescence transients. It was observed that the bound exciton peaks (DX and SDX) intensities saturated under higher excitation power. It could be due to low number of defects in the small volume of NWs.

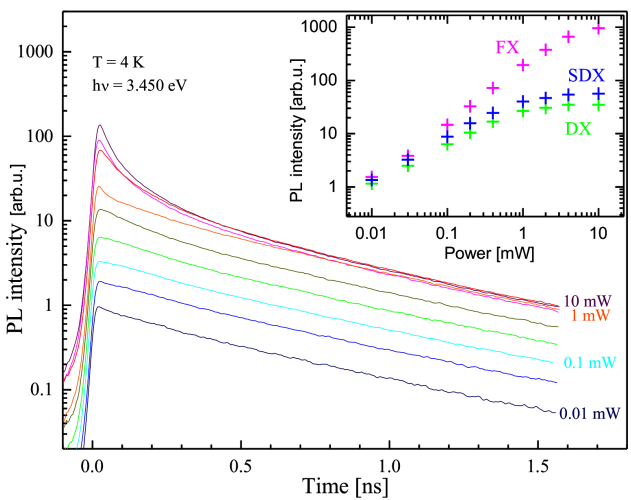

Fig. 3. Transients of the SDX under different excitation power. Inset: power dependences of three main luminescence peaks present in the GaN NWs.

The effect could be enhanced by reduction of active volume of a nanowire due to piezoelectric and spontaneous polarization of the GaN that generated strong electric field along the $C$ axis [8,9] (it is along the length of nanowire).

Numerical calculations of electric field and potential distributions for a GaN/Si NW (by the method described in [10]) shows that free electrons drift to GaN/Si interface screening the electric field. In this situation high charge concentration is expected at both ends of the NW. Only the central part of the NW is screened and can emit light. The excited particles can accumulate in this area which leads to the bound exciton saturation and the bi-exciton related effects.

Temperature dependent measurements showed that between 4 and $50 \mathrm{~K}$ the DX and SDX lifetimes decreased of about $30 \%$, with increase of temperature. On the other hand, the FX lifetime became longer, similar to the DX lifetime. This was probably due to release of excitons from the defects because simultaneously the DX and SDX intensities decreased. The activation energy for exciton escape was very low, about $1.5 \mathrm{meV}$. It was similar for DX and SDX. At temperature above $50 \mathrm{~K}$ all lifetimes became shorter and the emission energies shifted to lower values similarly like in homoepitaxial GaN [11]. 
PL transients of the GaN/Si NWs were not mono-exponential. At low temperature they can be fitted with a two-exponential decay. However, at temperatures of $50 \mathrm{~K}$ or above the best fit was obtained in a model assuming that recombination rate $\mathrm{d} n / \mathrm{d} t$ is proportional also to the square of concentration of particles $n^{2}$ (the Langevin recombination [12]):

$$
\mathrm{d} n=-\left(r_{1} n+r_{2} n^{2}\right) \mathrm{d} t,
$$

where $r_{1}, r_{2}$ - linear and quadratic recombination rates.

The solution of this equation can be written in a form

$$
n(t)=\frac{n_{0} \exp \left(-r_{1} t\right)}{1+n_{0}\left[\exp \left(-r_{1} t\right)-1\right] / N_{\mathrm{K}}},
$$

where $n_{0}=n(t=0), N_{\mathrm{K}}=r_{1} / r_{2}$ can be interpreted as key concentration weighting linear and quadratic term. The observed luminescence intensity is proportional to the number of recombining particles $\gamma \mathrm{d} n$ :

$$
I(t)=\gamma r_{1} n_{0} \exp \left(-r_{1} t\right) \frac{1+n_{0} / N_{\mathrm{K}}}{\left\{1+n_{0}\left[\exp \left(-r_{1} t\right)-1\right] / N_{\mathrm{K}}\right\}^{2}} .
$$
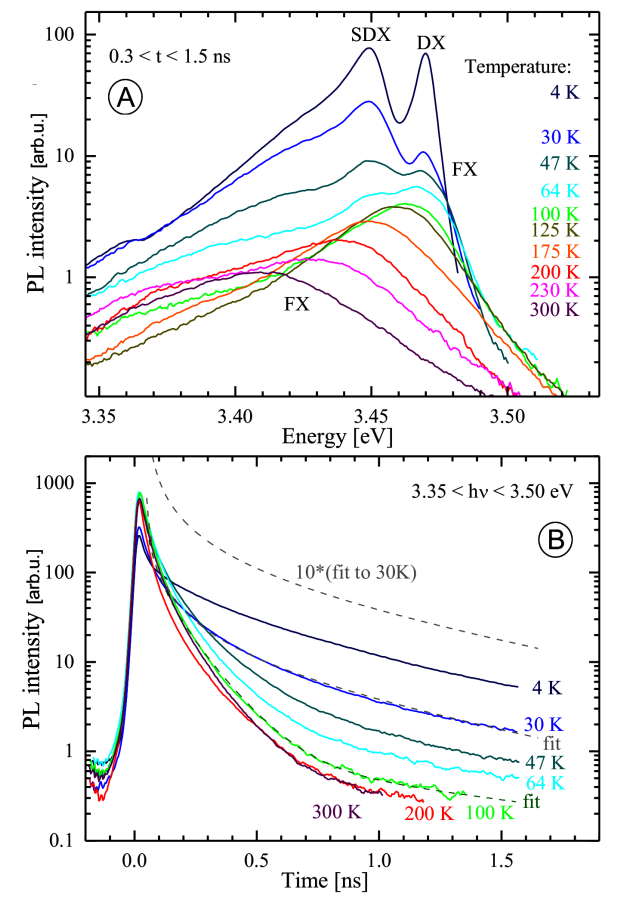

Fig. 4. Photoluminescence of NWs at different temperatures: (A) spectra, (B) transients. Dashed lines present fits of our model. The $30 \mathrm{~K}$ fit is shifted up to present the shape of the curve.

The curves calculated by Eq. (4) fit very well to the observed PL decays. Some of them (for $30 \mathrm{~K}$ and $100 \mathrm{~K}$ ) are plotted in Fig. 4. The curves fit so well that they are hard to distinguish from experimental data, so one of them $(30 \mathrm{~K})$ was shifted in Fig. 4 to show its shape. Single quadratic curve described by three parameters gives better fit than a two-exponential curve having four parameters. So we can conclude that the decay is influenced by bimolecular recombination.

\section{Conclusions}

Optical characterization of the NWs reveals bright emission, sharp peaks (2 meV) and long lifetimes (DX $0.4 \mathrm{~ns}$, SDX 0.6 ns). The DX peak is at the same position, $h \nu=3.472 \mathrm{eV}$, as in homoepitaxial GaN, which confirms good quality of the material.

The NW show some new features: long-lived peak (0.6 ns) at $3.45 \mathrm{eV}$ probably being due to surface defect bound excitons and a broad $(0.05 \mathrm{eV})$ band centered at $3.47 \mathrm{eV}$ caused probably by bi-exciton recombination. Precise analysis of this band by time-resolved PL revealed fast, non-exponential dynamics that can be described by bimolecular recombination model. The obtained agreement proves that the band is due to exciton-exciton interactions.

\section{Acknowledgments}

This work was partially supported within European Regional Development Fund, through grant Innovative Economy (POIG.01.01.02-00-008/08 NanoBiom) and by the JU ENIAC MERCURE project No. 120220.

\section{References}

[1] L. Geelhaar, C. Chèze, B. Jenichen, O. Brandt, C. Pfüller, S. Münch, R. Rothemund, S. Reitzenstein, A. Forchel, T. Kehagias, P. Komninou, G.P. Dimitrakopulos, T. Karakostas, L. Lari, P.R. Chalker, M.H. Gass, H.Riechert, IEEE J. Sel. Top. Quant. Electron. 17, 878 (2011).

[2] O. Brandt, C. Pfüller, C. Chèze, L. Geelhaar, H. Riechert, Phys. Rev. B 81, 045302 (2010).

[3] A. Das, P. Bhattacharya, A. Banerjee, M. Jankowski, Phys. Rev. B 85, 195321 (2012).

[4] R. Stępniewski, K.P. Korona, A. Wysmołek, J.M. Baranowski, K. Pakuła, M. Potemski, G. Martinez, I. Grzegory, S. Porowski, Phys. Rev. B 56, 15151 (1997).

[5] P. Corfdir, P. Lefebvre, J. Ristić, P. Valvin, E. Calleja, A. Trampert, J.-D. Ganière, B. Deveaud-Plédran, J. Appl. Phys. 105, 013113 (2009).

[6] M. Ueta, H. Kanzaki, K. Kobayashi, Y. Toyozawa, E. Hanamura, Excitonic Processes in Solids, Springer-Verlag, Berlin 1986.

[7] K.P. Korona, J. Kuhl, J.M. Baranowski, S. Porowski, Phys. Status Solidi B 216, 85 (1999).

[8] F. Bernardini, V. Fiorentini, D. Vanderbilt, Phys. Rev. B 56, R10024 (1997).

[9] O. Ambacher, J. Majewski, C. Miskys, A. Link, M. Hermann, M. Eickhoff, M. Stutzmann, F. Bernardini, V. Fiorentini, V. Tilak, B. Schaff, L.F. Eastman, J. Phys., Condens. Matter 14, 3399 (2002).

[10] K.P. Korona, A. Drabińska, P. Caban, W. Strupiński, J. Appl. Phys. 105, 083712 (2009).

[11] K.P. Korona, A. Wysmołek, K. Pakuła, R. Stępniewski, J.M. Baranowski, I. Grzegory, B. Eucznik, M. Wróblewski, S. Porowski, Appl. Phys. Lett. 69, 788 (1996).

[12] U. Albrecht, H. Bässler, Phys. Status Solidi B $\mathbf{1 9 1}$ 455 (1995). 